\title{
FINGERPRINT INDOOR POSITION SYSTEM BASED ON OPENMAC
}

\author{
A.Verónica Medina, José A. Gómez, Octavio Rivera, Enrique Dorronzoro, Manuel Merino \\ Departamento Tecnología Electrónica,Seville University, ETSI-INFORMÁTICA Avda. Reina Mercedes s/n, Seville, Spain \\ (vmedina,jgomezdte)@us.es,(octavio,enriquedz,manmermon)@dte.us.es
}

Keywords: IEEE 802.15.4, RSSI, Centroid, Indoor position, ZigBee, WSN

\begin{abstract}
This paper presents a work in progress for the developing of a fingerprint indoor position system based on OpenMAC, an IEEE 802.15.4 embedded software implementation from Atmel to be used in different scenarios like e health, ambient living or smart building. The system is thought to work as another one that we have yet developed but using the BitCloud Stack, a full-featured, second generation embedded software stack from the same supplier, but improving it. The first steps followed up in the system development are shown in this paper.
\end{abstract}

\section{INTRODUCTION}

WSNs (Wireless sensor Network) are present in many applications. They are used in Ambient Living (Hristova,2008), (Figueiredo, 2010), (Hong 2008), (Sun 2009) or Smart building (Martin, 2009), (Dietrich, 2010), (Chen, 2010), (Han, 2010), (Snoonian, 2003) researching fields for solving data acquisition process, depending on its applications, it can be used ambient or user sensors and actuators for making decisions. The knowledge of the subject's position it is very useful in this kind of systems because depending on it the decisions to be made are different.

As stated in (Konrad, 2005, 2006), a number of indoor location tracking systems have been proposed in the literature, based on RF signals, ultrasound, infrared, or some combination of modalities. Given a model of radio signal propagation in a building or other environment, received signal strength can be used to estimate the distance from a transmitter to a receiver, and thereby triangulate the position of a mobile node. However, this approach requires detailed models of RF propagation and does not account for variations in receiver sensitivity and orientation.
An alternative approach is to use empirical measurements of received radio signals, known as RSSI, Receiver Signal Strength Indicator, to estimate location. By recording a database of radio "signatures" along with their known locations, a mobile node can estimate its position by acquiring a signature and comparing it to the known signatures in the database, also known as fingerprints. A weighting scheme can be used to estimate location when multiple signatures are close to the acquired signature

All of these systems require that the signature database are collected manually prior to system installation, and rely on a central server (or the user's mobile node) to perform the location calculation. Several systems have demonstrated the viability of this approach, one of those is MoteTrack (Konrad 2005, 2006).

MoteTrack's basic location estimation uses a signature based approach that is largely similar to RADAR (Bahl, 2000) that obtains a 75th percentile location error of just under $5 \mathrm{~m}$, but decreased the location error by $1 / 3$ in MoteTrack.

We implemented (Medina, 2011) a system similar to MoteTrack, a signature-based localization scheme, but using other motes, Meshnetics' one (http://www.meshnetics.com/), that uses different 
RCB (MCU and transceiver) and, also, different software, i. e., the BitCloud Stack, a ZigBee PRO certified platform. That system was tested and had the same precision as MoteTrack, but it has some drawbacks that are going to be solved using the OpenMAC indeed of BitCloud Stack, both embedded software developed by Atmel(Atmel have acquired MeshNetics's ZigBee Intellectual Properties).

In Section 2 an overview of the system is presented. BitCloud Implementation is shown in Section 3. OpenMAC solution is explained in section 4. Conclusions are established in section 5 .

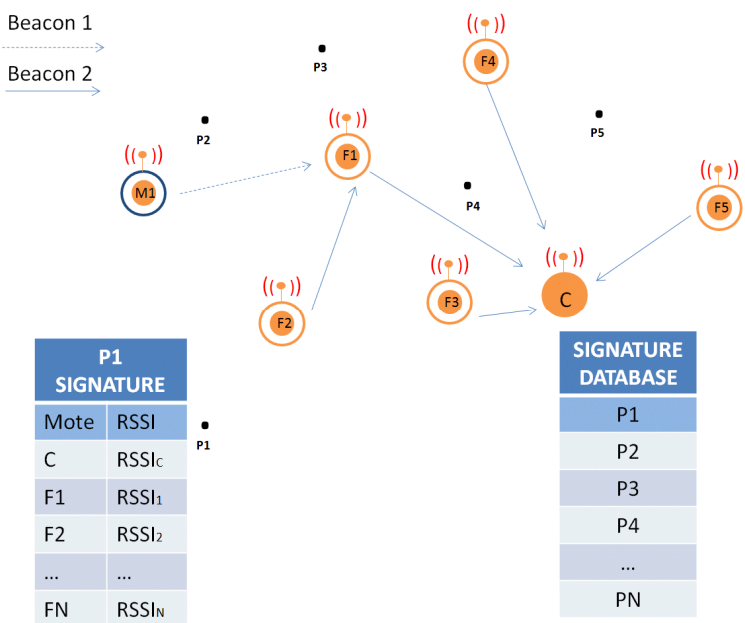

Figure 1: System Overview. M1 is a mobile node, F1-F5 are fixed nodes, and $\mathrm{C}$ is the coordinator, also a fixed node. M1 periodically sends a beacon message, beacon 1 , to inform the others node that is present, all fixed node that receives it, save the RSSI of that message in a table. Fixed node periodically sends a message to $\mathrm{C}$, beacon 2 , to inform about the RSSI that they receive from mobiles node, M1 in this case.

\section{SYSTEM OVERVIEW}

In this section we give an overview of the system, shown in Figure 1, that is based on low-power, embedded wireless devices, MeshNetics's sensor "motes". The advantages of this platform over other motes is that it's equipped with extra sensors and others could be easily connected to them if the application requires it, so for prototyping these motes work quite well. Other advantage of this mote is that the supplier has developed the ZigBee RFC4 stack architecture (ZigBee, 2009) in a software pack called BitCloud Stack and also the IEEE 802.15.4 in a software pack called OpenMAC.
In our system, a building or an area is populated with a number of MeshNetics's motes acting as fixed nodes, one of them acting as coordinator, $\mathrm{C}$. Fixed nodes send to $\mathrm{C}$ periodic beacon messages, beacon 2, which consist of an $n$-tuple of the format \{MobileID, RSSI\}, where $\mathrm{n}$ is the number of mobile nodes. MobileID is a unique identifier of a mobile node, and RSSI the value received from the last beacon message, beacon 1, received from that mobile node in a specific fixed node.

The location estimation problem consists of a two-phase process: an offline collection of reference signatures followed by online location estimation. As in other signature-based systems, the reference signature database is acquired manually by a user with a mobile node and a PC connected to C. Each reference signature, shown as black dots in Figure 1, consists of a set of signature tuples of the form \{sourceID, meanRSSI\}, where sourceID is the fixed node ID and meanRSSI is the mean RSSI of a set of beacon messages received over some time interval. Each signature is mapped to a known location by the user acquiring the signature database (P1-P5 in Figure 1).

\subsection{Location Estimation}

Given a mobile node's received signature, $\boldsymbol{s}$, received from the fixed nodes, and the reference signature set $\boldsymbol{R}$, the mobile node's location can be estimated as follows. The first step is to compute the signature distances, from $\boldsymbol{s}$ to each reference signature $\boldsymbol{r}_{\boldsymbol{i}} \boldsymbol{\epsilon} \boldsymbol{R}$. We employ the Manhattan distance metric,

$$
M(r, s)=\sum_{t \in T}|R S S I(t) r-R S S I(t) s|
$$

where $\boldsymbol{T}$ is the set of signatures tuples presented in both signature, $\boldsymbol{R S S I}(\boldsymbol{i}) \boldsymbol{r}$ is the RSSI value in the signature appearing in signature $r_{i}$ and $\boldsymbol{R S S I}(i) \boldsymbol{s}$ is the RSSI value in the signature appearing in signature $s$.

Given the set of signature distances, the location of a mobile node can be calculated in several ways. We consider the centroid of the set of signatures within some ratio of the nearest reference signature. Given a signature $s$, a set of reference signatures R, and the nearest signature $r^{*}=\operatorname{argminr} \epsilon \mathrm{R} M(r, s)$, we select all reference signatures $r \in R$ that satisfy

$$
\frac{M(r, s)}{M(r *, s)}<c
$$


for some constant c, empirically-determined. The geographic centroid of the locations of this subset of reference signatures is then taken as the mobile node's position. Small values of $\mathrm{c}$ work well, generally between 1.1 to 1.2 .

\section{BITCLOUD IMPLEMENTATION}

The BitCloud implementation was developed in a Meshbean development board. We have used this mote because they have leds, buttons, additional sensors and can easily be connected other sensors that can be used for different purpose applications of the indoor position system, ambient living and smart buildings, so for prototyping works quite well. They also have a USART accessible by a USB connector, so a PC can be connected via USB port, emulating it a COM port, for both programming and receiving information, in our case beacons and sensor values.

A MeshNetics's mote is shown in Figure 2, in this case, it has an integrated PCB antenna, but we have used others that aren't, this affects only the range of coverage. This mote has a MCU wireless, called ZigBit, a compact 802.15.4/ZigBee module featuring record-breaking range performance and exceptional ease of integration. It integrates both the ATmega1281 microcontroller and AT86RF212 transceiver of ATMEL (www.atmel.com) so the AVR tools are necessary for programming purposes.

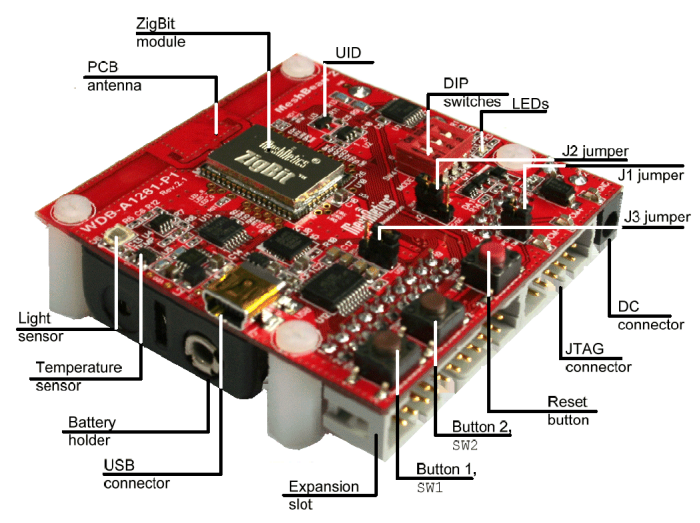

Figure 2: Meshbean development board.

In ZigBee there are three kinds of devices, each one having its own purpose:
1. Coordinator (C): A full function device (FFD) that it is in charge of creating the PAN (Personal Area Network) and typically is the point of the WSN to acquire all sensors information from all the other motes to be shown in a computer. The icon uses to represent this device is a filled circle, Figure 1 shown one.

2. Router (R): A FFD that it is in charge of routing when the range of coverage requires this capability, so it is possible to have dynamic topologies. The icon uses to represent this device is a small filled circle inside a circle, Figure 1 shown six ones.

3. End device (ED): A reduced function device (RFD) that is always slept (to reduce consumption) and only wakes up to do a specific task, for instance, to send sensor information to the WSN, typically directed towards $\mathrm{C}$. The icon use is a not filled circle, this is, like the $\mathrm{R}$ icon in Figure 1, but no filled circle inside (Figure 7).

So a ZigBee WSN is composed of one C, many EDs and many Rs. Each kind of devices can receive what the other transmit, if they are in the same range of coverage, because the transmission media is share by all one, but not all the information receive is processed (the explanation of why this is that way is out of the scope of this paper).

As explained in the previous section, to determinate position, we require to kinds of beacons, beacon1 and beacon2. Beacon 1 is used to inform other devices that a mobile mote is present and beacon 2 is used to inform $\mathrm{C}$ the RSSI value that a fixed mote receives from a mobile one for location estimation. To send both beacons in BitCloud Stack, we have to use the information saved in a table at the network layer called neighbor table. This table registered all the FFD, this is, motes that are $\mathrm{Co}$, that are in the range of coverage of a determinated mote and for each one it registers the RSSI value of the received signal from that mote. Periodically, a FFD device sends a MAC layer message to inform other that is in the PAN, so that message is used by neighbor motes to measure the RSSI value of the received signal and to save it in their own neighbor table. So beacon 1 is sent automatically by the protocol stack. As only FFD sends this kind of message the mobile motes have to be R, as shown in Figure 1 .

To send periodically beacon 2 messages, each fixed motes search in its neighbour table to find out if the mobile mote is in its range of coverage, if so, the beacon 2 is sent to $\mathrm{C}$ with the information required as explained in section 2. As neighbour table is only in FFD, fixed motes have also to be R. 


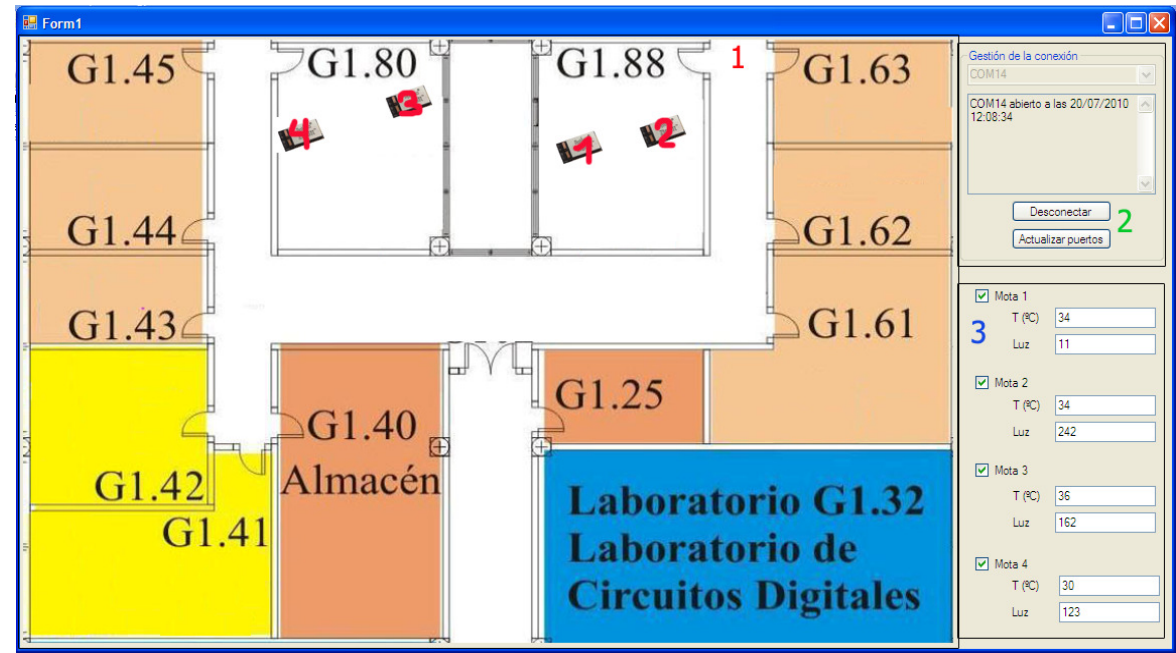

Figure 3: Position System Interface.

\begin{tabular}{|c|c|c|c|c|c|}
\hline Sre PAM & MAL Sre & Dest PAN & MaC Dest & MAC Seq Mo & Facket Type \\
\hline & & Dxfffi & Dxffef & $0 \times 04$ & Command: Beacon Request \\
\hline & & Dxfiffi & Dxffef & $0 \times 04$ & Command: Beacon Request \\
\hline Dxdada & $0 \times 0000$ & & & $0 \times 05$ & Beacon: BO: $15,50: 15$, PC: 1 , मP: 1 \\
\hline \multirow[t]{6}{*}{ oxfffi } & $0 \times 0000000000000002$ & Oxdada & $0 \times 0000$ & $0 \times 0.5$ & Cominand: Association Request \\
\hline & & & & $0 \times 0.5$ & Acknowledgment \\
\hline & $0 \times 0000000000000002$ & Dxdada & $0 \times 0000$ & $0 \times 06$ & Comimand: Data Request \\
\hline & & & & $0 \times 06$ & Acknowledgment \\
\hline & $0 \times 0000000000000000$ & Dxdada & $0 \times 0000000000000002$ & $0 \times 06$ & Comimand: Association Response \\
\hline & & OXEFẼ & DXẼẼ & $0 \times 06$ & $\begin{array}{l}\text { ACknowledgment } \\
\text { Cominand: Bisacon Recosest }\end{array}$ \\
\hline Oxdada & $0 \times 0002$ & & & $0 \times 07$ & Beacon: B0: 15, 50: 15, PC: 0, ДP: \\
\hline & $0 \times 0000$ & & & $0 \times 07$ & Beacon: BO: 15, 50: 15, PC: 1 , AP: 1 \\
\hline \multirow{7}{*}{ oxfeff } & 0x0000000000000001 & Dxdada & $0 \times 0000$ & $0 \times 05$ & Command: Association Recuest \\
\hline & & & & $0 \times 0.5$ & Acknowledgment \\
\hline & $0 \times 0000000000000001$ & Oxdada & $0 \times 0000$ & $0 \times 06$ & Command: Data Request. \\
\hline & & & & $0 \times 06$ & Acknowledgment \\
\hline & $0 \times 0000000000000000$ & Dxdada & 0x0000000000000001 & $0 \times 08$ & Comimand Association Response \\
\hline & & & & $0 \times 08$ & Acknowledgment \\
\hline & & Dxffiff & Dxffef & $0 \times 04$ & Command: Beacon Request \\
\hline Dxdada & $0 \times 0002$ & & & $0 \times 08$ & Beacon: BO: $15,50: 15$, PC: 0, AP: 1 \\
\hline Oxdada & $0 \times 0001$ & & & $0 \times 07$ & Beacon: BO: 15, s0: 15, PC: 0, AP: 1 \\
\hline \multirow[t]{2}{*}{ Oxdada } & $0 \times 0000$ & & & $0 \times 09$ & Beacon: BO: $15,50: 15, \mathrm{PC}: 1, \mathrm{AP}: 1$ \\
\hline & & OXIFEFE & OXIEEE & $0 \times 0.5$ & Comimand: Beacon Request \\
\hline Oxdada & 0x0001 & & & $0 x 08$ & Beacon: BO: 15, S0: 15, PC: 0, AP: 1 \\
\hline Oxdada & $0 x 0000$ & & & 0x0a & Beacon: BO: 15, S0: 15, PC: 1 , AP: 1 \\
\hline Oxdada & $0 \times 0002$ & & & $0 \times 09$ & Beacon: BO: 15, S0: 15, PC: 0 , AP: 1 \\
\hline \multirow[t]{2}{*}{ oxeffe } & $0 \times 0000000000000003$ & Dxclada & $0 \times 0002$ & $0 \times 06$ & Command: Association Recuest \\
\hline & $0 \times 0000000000000003$ & Dxdada & $0 \times 0002$ & $\begin{array}{l}0 \times 06 \\
0 \times 07\end{array}$ & $\begin{array}{l}\text { Acknowledgment } \\
\text { Cominand: Data Reolest }\end{array}$ \\
\hline
\end{tabular}

Figure 4: Dantree Network Sensor Analyzer Output.

We deployed BitCloud solution over half floor of our Department Area, measuring roughly $225 \mathrm{~m}$. To cover all this area we required 7 fixed motes strategically placed. An off-line phase was required to fill in the signature database, once it was full, the system was ready to be tested.

Figure 3 shows the PC interface to show mobile mote position, four in this case. It also shows the mobile mote sensor information.

Although results were as expected as shown in (Medina, 2011), this solution has two drawbacks, in order to fix it correctly:
1. The mobile node has to be FFD so the power consumption is very high and it is a problem because mobile node is battery power.

2. The periodicity of beacon 1 messages can't be controlled as it is a MAC parameter not accessible by BitCloud Stack.

\section{OPEMAC IMPLEMENTATION}

OpenMAC is an open source implementation of IEEE802.15.4 Media Access Control (MAC) layer. 


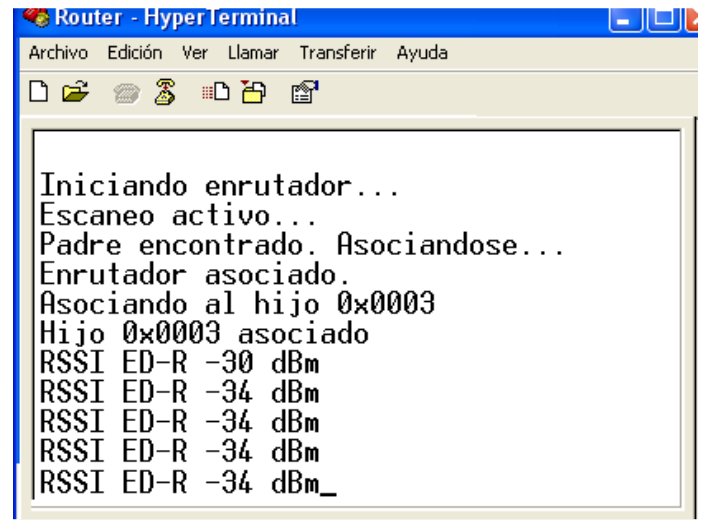

Figure 5: Hyperterminal Output, Beacon 1 Message.

It has a series of advantage over using BitCloud Stack:

1. Enables users, who do not require full functionality of BitCloud Stack, to develop custom WSN applications.

2. Enabled advanced users to modify OpenMAC internals to suit specific application needs.

3. Jump start application development on top of MAC with thoroughly documented sample applications.

3. Provide a convenient C API to developers not familiar with TinyOS or nesC programming language (technologies at the core of OpenMAC).

4. Provide a reference design to be ported to analogous hardware platforms.

To deploy the same indoor solution as in BitCloud Stack, it is necessary to create a PAN like the one shown in Figure 1, where there are all the kinds of ZigBee devices, C, R and ED and all implement the same functionally (FFD $\mathrm{C}$ and $\mathrm{R}$, RFD ED). So, $C$ creates the PAN and the other devices connect to the PAN. All communication flow is towards the $\mathrm{C}$, so $\mathrm{R}$ is in charge of forwarding packed when other Rs or EDs required, they are closer to it than to $\mathrm{C}$. R and ED connect to the PAN via $\mathrm{C}$ or other $\mathrm{R}$ that is already in the PAN.

To create the PAN, the MAC services implemented in OpenMAC for doing so, has been used. There is a bug in the OpenMAC software that couldn't be solved. When a R or ED tries to connect to a PAN, it can decide which device is going to be its father basing its decision in the RSSI of the beacon frame, a MAC layer service. As shown in Figure 4, although there are more than one device that send the beacon frame when it is requested, the OpenMAC software only offers as father, the one that sent the last, but that couldn't be the best one. In the lab test, this is not a problem, because all mote are close, but maybe it will be a problem when we will deploy the fixed mote, this is something that will be tested.

The two drawbacks of BitCloud are solved this way. In OpenMAC solution, mobile motes are EDs not R, so they are slept all the time and are only woken up when they have to send beacon 1 message, we control the frequency of transmission. This message is broadcasted, so all its neighbours are able to save the RSSI value of the received message, this information is required to inform $\mathrm{C}$ after. Figure 5 shows the output of a $\mathrm{R}$ that is receiving Beacon 1 messages from an ED (mobile mote), they are shown as "RSSI ED-R".

Periodically, $\mathrm{R}$ sends beacon 2 message to $\mathrm{C}$. This message is unicast, so a $\mathrm{R}$ that receives one, has to forward it, if the source $\mathrm{R}$ is one of its child. Figure 6 shows the output of $\mathrm{C}$ that is receiving Beacon 2 message from a $\mathrm{R}$ (fixed mote), they are shown as "RSSI ED-X", where $\mathrm{X}$ is $\mathrm{C}$ or $\mathrm{R}$ depending on the sender.

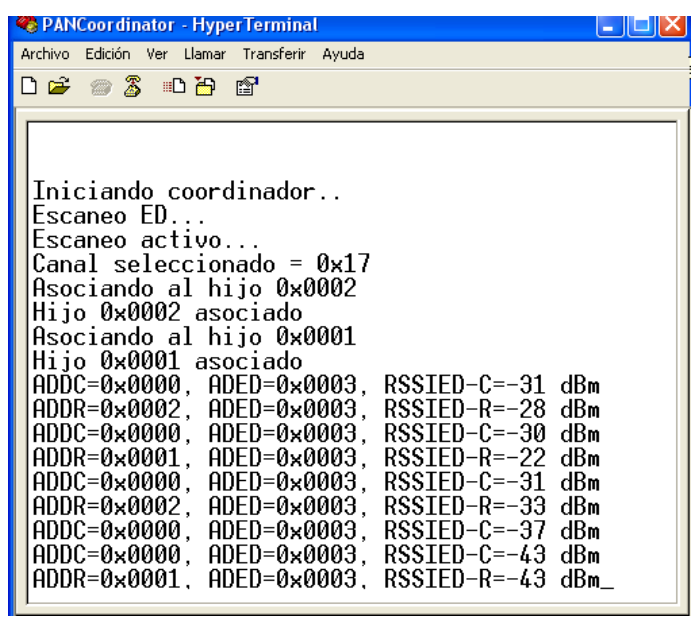

Figure 6: Hyperterminal Output, Beacon 2 Message.

Different scenarios (topologies) has been tested (Figure 7) in order to prove that the PAN works correctly and is set to deploy it over the half flour of our Department. So the following work is to do the same steps as we did with BitCloud Stack.

\section{CONCLUSIONS}

The PAN infrastructure for an indoor position system based on fingerprints is developed using OpenMAC in a lab environment. The two beacon messages required for the position estimation has been implemented. Next step will be to reuse all the source code implemented in the PC for BitCloud 


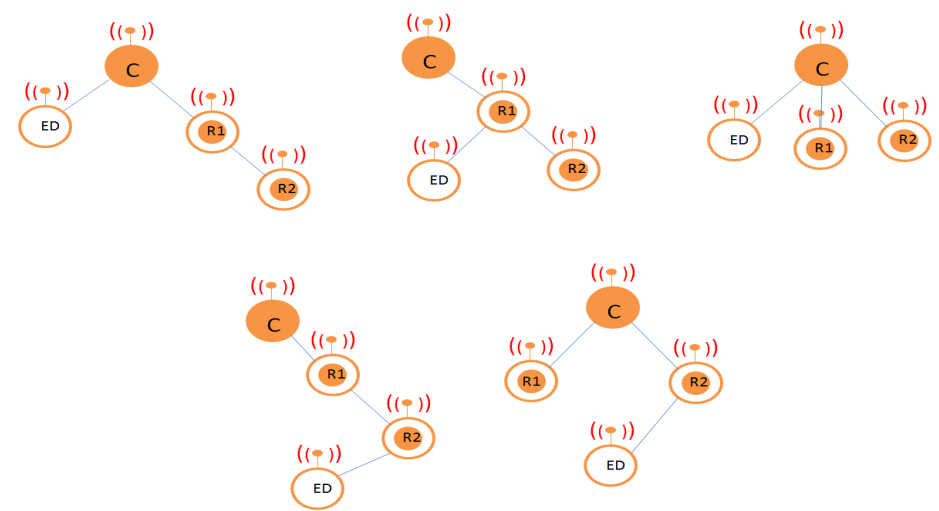

Figure 7. Topologies Tested. The blue lines shows node's father.

positioning solution. For doing so some setting has to be done in other to send the same way the information from the PAN towards the PC connected to the C. Once solve, an off-line phase is required to fill in the signature database. Then the system will be ready to be fixed and tested.

\section{ACKNOWLEDGEMENTS}

This work has been carried out within the framework of two research programs: (P08-TIC3631) - Multimodal Wireless interface (IMI) funded by the Regional Government of Andalusia and Efficient and Health Intelligent Technologies Oriented to Health and comfort in Interior Environments (TECNO-CAI) approved project at the fifth call of CENIT program by the Innovation Science Ministry of Spain (CDTI and Ingenio 2010 Program).

\section{REFERENCES}

Hristova, A.; Bernardos, A.M.; Casar, J.R. 2008. Contextaware services for ambient assisted living: A casestudy. Applied Sciences on Biomedical and Communication Technologies, 2008. ISABEL '08. First International Symposium on.

Figueiredo, C.P.; Gama, O.S.; Pereira, C.M.; Mendes, P.M.; Silva, S.; Domingues, L.; Hoffmann, K.-P. 2010 Autonomy Suitability of Wireless Modules for Ambient Assisted Living Applications: WiFi, ZigBee, and Proprietary Devices. Sensor Technologies and Applications (SENSORCOMM).

Hong Sun; De Florio, V.; Ning Gui; Blondia, C., Towards. 2008. Building Virtual Community for Ambient Assisted Living. Parallel, Distributed and NetworkBased Processing. 16th Euromicro Conference on.
Sun, H.; De Florio, V.; Gui, N.; Blondia, C., 2009. PRomises and Challenges of Ambient Assisted Living Systems. Information Technology: New Generations, 2009. ITNG '09. Sixth International Conference on. Page(s): 1201 - 1207.

Martin, H.; Bernardos, A.M.; Bergesio, L.; Tarrio, P., 2009. Analysis of key aspects to manage wireless sensor networks in ambient assisted living environments. Applied Sciences in Biomedical and Communication Technologies, 2009. ISABEL 2009. 2nd International Symposium. Page(s): 1 - 8.

Dietrich, D.; Bruckner, D.; Zucker, G.; Palensky, P. 2010. Communication and Computation in Buildings: A Short Introduction and Overview. Volume: 57 , Issue:.

Chen, Po-Wei; Ou, Kuang-Shun; Chen, Kuo-Shen. 2010. IR indoor localization and wireless transmission for motion control in smart building applications based on Wiimote technology. SICE Annual Conference 2010.

Han Chen; Chou, P.; Duri, S.; Hui Lei; Reason, J. 2009. The Design and Implementation of a Smart Building Control . Page(s): 255 - 262.

Snoonian, D, Smart buildings. 2003. Spectrum, IEEE Volume: 40 , Issue: 8 Digital Object Identifier: 10.1109/MSPEC. Page(s): 18 - 23.

Bahl P., Padmanabhan VN. 2000. RADAR: an in-building RF-based user location and tracking system. In: INFOCOM, pp 775-784.

Konrad L. and Matt W. 2005. MoteTrack: A Robust, Decentralized Approach to RF-Based Location Tracking. In Proceedings of the International Workshop on Location and Context-Awareness (LoCA 2005) at Pervasive May 2005.

Konrad L., Matt W. 2006. MoteTrack: A Robust, Decentralized Approach to RF-Based Location Tracking. To Appear in Springer Personal and Ubiquitous Computing, Special Issue on Location and Context-Awareness. . ISSN: 1617-4909 (Print.

Medina A. V., Gómez I., Romera M., Gómez J.A. and Dorronzoro E. 2011. Indoor Position System based on BitCloud Stack for Ambient Living and Smart Buildings. In 3rd International ICST Conference on IT Revolutions. Córdoba, Spain.

ZigBee, 2009 RF4CE Specification. Version 1.00. ZigBee Document 094945r00ZB, March 17th, 2009. 